\title{
Nonlinear Parametric Model Identification and Model Based Control of S. cerevisiae Production
}

\author{
Bulent Akay \\ Ankara University, Faculty of Engineering, Department of Chemical Engineering, \\ 06100 Tandogan,Ankara,Turkey \\ bakay@eng.ankara.edu.tr
}

\begin{abstract}
This work shows the application of Parametric Model Identification method which is Recursive Least Square for the estimation of nonlinear model parameters. The model of the bioreactor dynamics was assumed in the form of regression model. DMC (Dynamic Matrix Control) and PID Control systems of S.cerevisiae yeast production in a batch bioreactor have been investigated experimentally to achieve the optimal operating condition. The performance of these control algorithms was compared with each other.
\end{abstract}

\section{Introduction}

In many cases the processes are so complex that it is not possible to obtain reasonable models using only physical insight. In such cases one is forced to use identification techniques. However, this technique needs an appropriate model structure.

DMC has provided researchers with a powerful tool for dynamic optimization and control for process industry. These control systems employing linear models include DMC [1] and simplified MPC. Recently there has been an increasing interest in the use of DMC for controlling plant-wide control [2,3].

In this study, a bioreactor which produces S.cerevisiae is modelled by parametric nonlinear models. Several identification techniques were used to define the model parameters. Comparison general nonlinear NARMAX model and experimental data from related system was demonstrated. Identified model was used to find the system matrix for DMC control.

\section{Nonlinear Model Parametric Identification}

A polynomial NARMAX model was constructed for design of nonlinear controller system . The general, discrete-time NARMAX Model given by eq. (1):

$y(k+1)=f\left(y(k), \ldots \ldots, y\left(k-n_{y}\right), U(k), \ldots . ., U\left(k-n_{u}\right), e(k), \ldots ., e\left(k-n_{c}\right)\right.$ 
Square wave signal has been used in the identification of nonlinear system. The model parameters have been evaluated by using Recursive Least Square Method. NARMAX model which is used in this study was given below:

$$
y(k)=-a_{1} y(k-1)-a_{2} y(k-2)+b_{0} u^{0.78}(k-1)+e(k)
$$

Model parameters were calculated as $a_{1}=-0.5408, a_{2}=-0.4641, b_{0}=0.0339$.

\section{Model Based Control}

In DMC, the manipulated variable is adjusted previously and the controlled variable is influenced by these adjustments as well as disturbances. To minimize performance index $\mathrm{J}$, the sum of the error squared is calculated

$$
J=\sum_{i=1}^{N P}\left[X^{s e t}(k+i)-X_{n e w}(k+i)\right]^{2}+f_{t} \sum_{i=1}^{N C}[\Delta u(k+i)]^{2}
$$

Where $\mathrm{X}_{\text {new }}$ is the closed-loop real response for the value at the $\mathrm{i}$ th step into the future and calculate by using eq. 4 .

$$
X_{n e w}=X_{\text {past }}+\underline{A \Delta u}+\underline{-}
$$

Here NP denotes the future time over which the control performance is evaluated and termed the output horizon. $\mathrm{NC}$ is the number of adjustments and is given by the input horizon. NC must be less than NP.

$A$ is the dynamic matrix that is composed of the step response coefficients. $X^{\text {set }}$ is the set point and can remain constant at its current value in the future. The goal of perfect controlled-variable performance would be to have zero error for all samples in the future. The solution to this problem is a least-square solution in the form of the following control increments:

$$
\underline{\Delta u}=\left[\underline{A}^{T} \underline{A}+f_{t}^{2} \underline{I}\right] \underline{A}\left(X^{\text {set }}-X_{\text {past }}-\underline{d}\right)
$$

\section{Control Results}

The parameters necessary for the DMC and PID algorithms were found from the response curve obtained by performing simple open-loop step tests (Figure 1). As can be seen from this figure the agreement between the response obtained from the computer simulation program and experimental data is sufficiently good. Dynamic Matrix was calculated from this reaction curve and given below. 


$$
A=\left[\begin{array}{cccc}
30.9 & 0 & 0 & 0 \\
30.7 & 30.9 & 0 & 0 \\
31.2 & 30.7 & 30.9 & 0 \\
31.4 & 31.2 & 30.7 & 0
\end{array}\right]
$$

The optimum values of the coefficients of PID controller were found using Cohen-Coon Method [4] as $K_{C}=68.6, \tau_{I}=2.4 \mathrm{~min}$ and $\tau_{D}=0.36 \mathrm{~min}$. The process model was chosen as first order dead time process. Dead time and open-loop process time constant were found as $0.5 \mathrm{~min}$ and $30.5 \mathrm{~min}$, respectively.

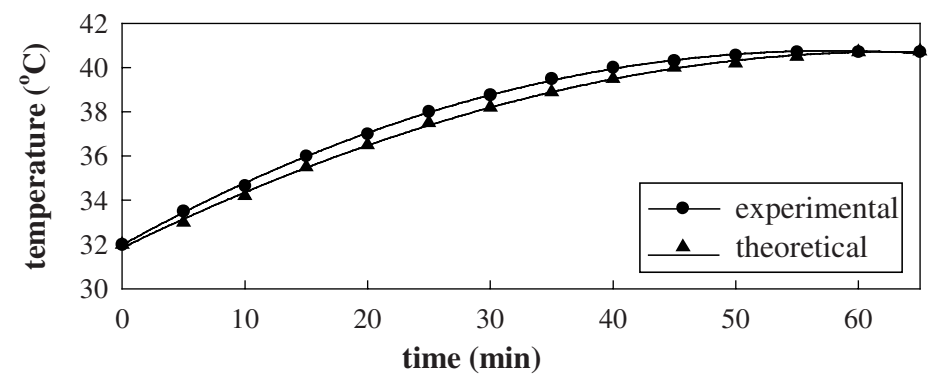

Fig. 1. Open loop temperature response

The selected DMC coefficients (NP, NC and f) were adjusted by a least-square technique applied to the error between observation and prediction. They are found as $\mathrm{NP}=4, \mathrm{NC}=1$ and $\mathrm{f}=0.1$. Time variations of the bioreactor temperature controlled by under DMC system in the experimental and theoretical studies are shown in Figure 2.

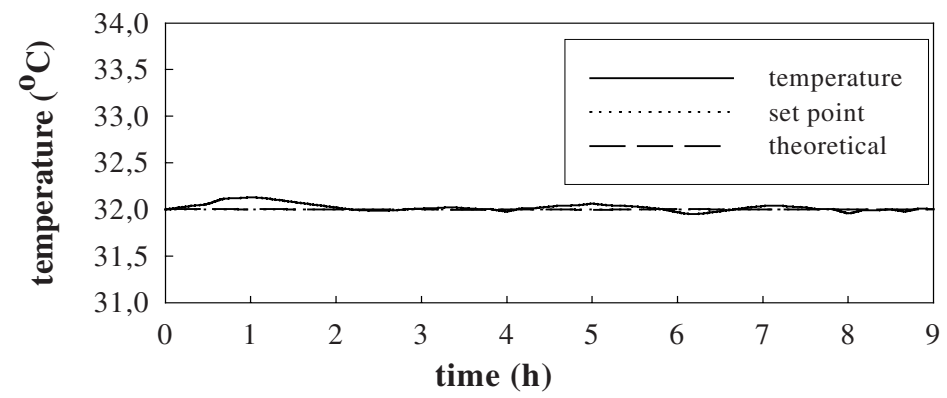

Fig. 2. Experimental and theoretical DMC of the bioreactor temperature

For the same condition, the PID control is applied to keep the bioreactor temperature at the desired set point. These experimental and theoretical time variations of the bioreactor temperature are given in Figure 3. In comparison with Figure 2, Figure 3 
shows that temperature moves around the desired trajectory at a higher frequency with some offset.

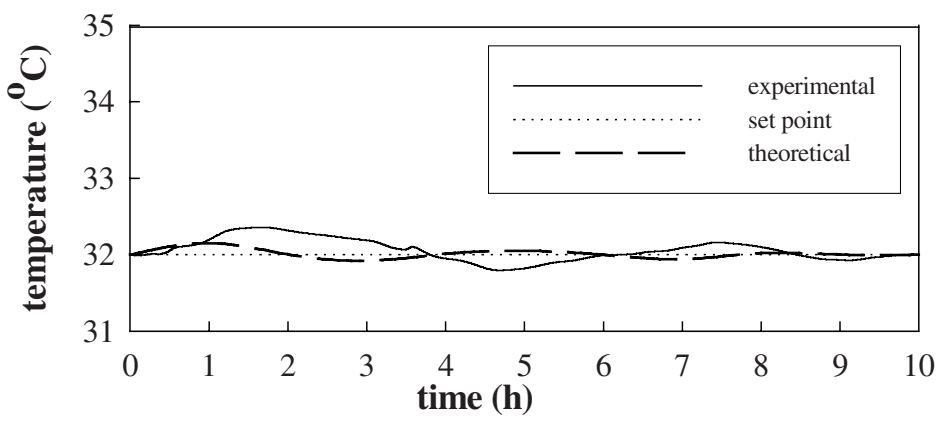

Fig. 3. Experimental and theoretical PID control of the bioreactor temperature

\section{Discussion}

The performance of DMC control was compared with the PID controller at the optimal set point in a batch bioreactor. This system was chosen because of its industrial importance and its nonlinearities. The oscillations of the temperature around the optimal path in the experiments were unavoidable. On the other hand, simulation results show excellent performance for DMC and PID controllers. Although there is no significant improvement in the experimental DMC results, it was seen that DMC provides a satisfactory control at least equal to a well-tuned PID controller.

Acknowledgement. This research has been supported by Ankara University Biotechnology Institute (Grant No. 29).

\section{References}

1. Cutler, C.R.: Dynamic matrix control: An optimal multivariable control algorithm with constraints, Ph.D. Thesis, University of Huston (1983)

2. Desphande, P.B. and Arulalan, G.R.: Simplified model predictive control, Ind. Eng. Chem. Res., Vol. 26. , (1987) 347-357.

3. Seborg, D.E., Edgar, T.F. and Mellichamp, D.A.: Process dynamics and control, JohnWiley and Sons, New York (1989)

4. Omstead D. R.: Computer Control of Fermentation Processes CRC Press, Inc., Boca Raton, Florida (2000) 